\title{
Índice de qualidade de efluente tratado em rampas de escoamento superficial
}

\author{
Quality index of wastewater treated in overland flow systems
}

\author{
Ana Paula Almeida Bertossi'* (), Larissa Cabral Milen ${ }^{2} \mathbb{1}$, \\ Giovanni de Oliveira Garcia² (i), Edvaldo Fialho dos Reis ${ }^{2}$ (1)
}

\section{RESUMO}

Com o objetivo de avaliar a qualidade do efluente doméstico tratado utilizando a técnica do escoamento superficial em rampas cultivadas com forrageiras, por meio da proposta de um índice de qualidade (IQ) adaptado para fertirrigação, e agrupar os efluentes quanto à similaridade das características químicas utilizando estatística multivariada, foi realizado um experimento montado em um delineamento inteiramente casualizado e no esquema de parcela subdividida $3 \times$ 2 com 5 repetições. As parcelas foram compostas de três espécies de forrageiras: aveia preta, azevém e centeio e as subparcelas formadas por duas taxas de efluente aplicadas no tempo: 50 e 100 kg.ha'.1ia' de demanda bioquímica de oxigênio (DBO). O IQ foi desenvolvido utilizando a análise de componentes principais (ACP), que proporcionou a redução das 25 características analisadas nos efluentes em duas componentes que explicaram 90,54\% da variância total. O IQ para os efluentes tratados apresentou de alta a baixa restrição ao uso na fertirrigação e o efluente bruto demonstrou restrição severa, já a análise de agrupamento formou três grupos distintos de qualidade, que diferiram quanto à taxa de aplicação de efluente utilizada no sistema de tratamento.

Palavras-chave: estatística multivariada; esgoto doméstico; tratamento.

\begin{abstract}
The objective of this study was to evaluate the quality of treated domestic effluent using the surface runoff technique in slopes cultivated with forages. A quality index was adapted for fertigation and to group effluents that were similar in chemical characteristics using multivariate statistics. The experiment consists of a completely randomized design with a $3 \times 2$ subdivided plot scheme with 5 replicates. The plots had three forage species: black oat, ryegrass and rye and the subplots had two effluent rates applied in time: 50 and $100 \mathrm{~kg} \mathrm{ha}^{-1} \mathrm{day}^{-1}$ of BOD. The quality index was developed using the main components analysis, which provided a reduction of the 25 characteristics analyzed in the effluents in two components that explained $90.54 \%$ of the total variance. The quality index for the treated effluents presented high to low restriction in the fertigation and the raw effluent presented severe restriction. The cluster analysis formed three distinct groups of quality, which differed in the effluent application rate used in the treatment system.
\end{abstract}

Keywords: Multivariate statistics; domestic sewage; treatment.

\section{INTRODUÇÃO}

No Brasil, a partir da década de 1980, as universidades, em parceria com algumas empresas de saneamento básico, iniciaram a implantação de estações de tratamento de esgoto por meio do escoamento superficial no solo em diferentes regiões do país, como alternativa de saneamento de baixo custo.

O escoamento superficial no solo proporciona a redução dos poluentes contidos no efluente por mecanismos físicos, químicos e biológicos. Nesse processo de tratamento, o efluente é aplicado, de forma controlada, na cabeceira de uma superfície vegetada plana e uniforme, com declividade variando de 2 a $8 \%$, ficando sujeito ao escoamento na superfície do solo, condição que possibilita sua depuração ao longo da rampa de tratamento. A vegetação absorve parte dos nutrientes contidos no efluente, promove proteção do solo contra erosão e favorece o desenvolvimento do biofilme, camada na qual os microrganismos se estabelecerão. A matéria orgânica é oxidada por esses microrganismos, e os sólidos suspensos são filtrados devido às propriedades de adsorção química e física do solo. Parte da água contida no esgoto percola pelo terreno ou evapora, e o restante é coletado em canaletas ao fim da rampa (TONETTI; COURACCI FILHO; STEFANUTTI, 2012).

Visto que, em certas regiões do país, a baixa precipitação pluviométrica não é suficiente para suprir a necessidade hídrica de determinados cultivos, um destino interessante para a água residuária resultante do escoamento superficial seria a fertirrigação (COSTA; BARROS JÚNIOR, 
2005). Apesar de apresentar efeitos benéficos, a utilização excessiva dessas águas na agricultura sem uma análise precisa de sua qualidade pode causar impactos negativos ao meio ambiente, à produtividade das culturas e à saúde de produtores e consumidores devido às quantidades de sais, nutrientes e patógenos presentes (HURLEY; SADIQ; MAZUMDER, 2012).

Dessa forma, o aspecto mais importante na utilização de águas residuárias para uso agrícola é o estabelecimento dos requisitos mínimos de qualidade que esta deve apresentar, e os parâmetros analisados devem estar relacionados com seus possíveis efeitos no solo, na cultura e no manejo da irrigação (BERNARDO; SOARES; MANTOVANI, 2006).

Para caracterizar uma água, são determinados diversos parâmetros, que, resumidos em um único valor numérico, indicam sua qualidade. Várias técnicas para elaboração de IQ de água têm sido usadas, sendo a mais empregada aquela desenvolvida pela National Sanitation Foundation Institution que avalia a qualidade da água para consumo humano (FLORES, 2002).

No entanto, o desenvolvimento de um índice global de qualidade de águas residuárias destinadas à fertirrigação seria uma alternativa viável para evitar grandes investimentos econômicos e temporais necessários na análise individual de um grande número de variáveis em águas residuárias, além de resumir vários dados em uma única expressão que facilite a tomada de decisão quanto ao uso dessas águas e ao controle de possíveis problemas causados ao solo e às plantas irrigadas (WANDA; GULULA; PHIRI, 2012; ARDILA; SALDARRIAGA, 2013).

Nesse sentido, objetivou-se, com este trabalho, avaliar a qualidade do efluente tratado pela técnica do escoamento superficial em rampas cultivadas com forrageiras, por meio da proposta de um IQ adaptado para fertirrigação a fim de evitar problemas de salinidade e sodicidade no solo e agrupar os efluentes quanto à similaridade das características químicas utilizando técnicas de estatística multivariada (análises de componentes principais - $\mathrm{ACP}-\mathrm{e}$ de agrupamento hierárquico de cluster - AAHC).

\section{MATERIAIS E MÉTODOS}

O experimento foi conduzido na área experimental do Centro de Ciências Agrárias da Universidade Federal do Espírito Santo em Alegre (ES), localizada sob as coordenadas de latitude $20^{\circ} 45^{\prime} 2,3^{\prime \prime}$ Sul, longitude 41²9'17,7" Oeste e altitude de 119 metros. O clima típico da região é quente e úmido no verão e seco no inverno, com temperatura anual média de $23,1^{\circ} \mathrm{C}$ e precipitação em torno de $1.200 \mathrm{~mm}$.
As unidades experimentais foram compostas de 15 calhas de fibrocimento com dimensões de $3 \times 0,9 \times 0,25 \mathrm{~m}$ (comprimento, largura $\mathrm{e}$ profundidade), as quais foram dispostas a uma declividade de aproximadamente $5 \%$ e preenchidas com solo retirado de um perfil natural de um latossolo vermelho-amarelo. Após coletado, o solo foi seco ao ar, peneirado em malha de $4 \mathrm{~mm}$ e depositado em igual massa em cada unidade experimental. Uma amostra foi coletada e encaminhada ao laboratório para caracterização química conforme metodologia proposta pela Empresa Brasileira de Pesquisa Agropecuária (Embrapa, 2009) (Tabela 1).

As metodologias de análise foram: $\mathrm{P}, \mathrm{K}, \mathrm{Na}, \mathrm{Fe}, \mathrm{Zn}, \mathrm{Cu}$ e $\mathrm{Mn}$ : extrator Mehlich; $\mathrm{pH} \mathrm{H} \mathrm{H}_{2} \mathrm{O}$ 1:2,5; $\mathrm{MO}$ : oxidação com $\mathrm{Na}_{2} \mathrm{Cr}_{2} \mathrm{O}_{7} \cdot 2 \mathrm{H}_{2} \mathrm{O}$ $+\mathrm{H}_{2} \mathrm{SO}_{4} 10$ mol.L $\mathrm{L}^{-1}$; Ca, $\mathrm{Mg}$ e Al: extrator $\mathrm{KCl} 1 \mathrm{~mol} . \mathrm{L}^{-1}$; e B: extrator água quente.

O experimento foi montado num delineamento inteiramente casualizado no esquema de parcela subdividida $3 \times 2$ com 5 repetições. As parcelas foram compostas de três espécies de forrageiras destinadas ao cultivo de outono/inverno - aveia preta (Avena strigosa), azevém (Lolium multiflorum) e centeio (Secale cereale) - e as subparcelas eram formadas por duas taxas de efluente aplicadas no tempo: 50 e $100 \mathrm{~kg} \cdot \mathrm{ha}^{-1} \cdot \mathrm{dia}^{-1}$ de demanda bioquímica de oxigênio (DBO). Cada taxa foi aplicada por um período de 28 dias.

O plantio das forrageiras foi feito diretamente nas unidades experimentais, as quais receberam adubação mineral conforme exigência nutricional e disponibilidade de nutrientes no solo. As sementes foram semeadas de maneira que cobrissem toda a superfície das calhas, a fim de promover a mesma uniformidade de plantas em todas as unidades experimentais. Após o plantio, elas foram irrigadas com água de abastecimento interno até o seu completo estabelecimento no solo. Após esse período, foi efetuado um corte de uniformização das plantas a $5 \mathrm{~cm}$ de altura do solo e foi iniciada a aplicação do esgoto doméstico de forma que satisfizesse as quantidades determinadas para cada tratamento.

O efluente doméstico utilizado no experimento, proveniente de fossas sépticas, era aplicado por gravidade na parte superior das parcelas experimentais por meio de tubulações perfuradas por um período de seis horas diárias de segunda a sexta-feira. O efluente gerado após o escoamento na rampa era coletado diariamente e, ao fim da aplicação de cada taxa, essas amostras foram misturadas, formando uma amostra composta e representativa de cada taxa. As amostras de efluente tratado e uma do efluente doméstico bruto (EDB) aplicado no sistema foram conduzidas ao laboratório para determinação de $\mathrm{pH}$, condutividade

Tabela 1 - Caracterização química do solo utilizado no experimento.

\begin{tabular}{|c|c|c|c|c|c|c|c|c|c|c|c|c|}
\hline $\mathrm{pH}$ & $P$ & $\mathrm{~K}$ & $\mathrm{Na}$ & $\mathrm{Ca}$ & $\mathrm{Mg}$ & $\mathrm{Al}$ & MO & $\mathrm{Zn}$ & $\mathrm{Fe}$ & $\mathrm{Mn}$ & $\mathrm{Cu}$ & B \\
\hline $\mathrm{H}_{2} \mathrm{O}$ & \multicolumn{3}{|c|}{-------mg.dm-3------ } & \multicolumn{3}{|c|}{------cmol $\mathrm{cdm}^{-3}$} & dag. $\mathrm{kg}^{-1}$ & \multicolumn{5}{|c|}{ 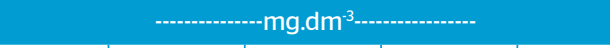 } \\
\hline 5,40 & 3,30 & 101,00 & 0,00 & 1,30 & 0,50 & 0,00 & 1,86 & 5,50 & 127,40 & 77,20 & 2,00 & 0,30 \\
\hline
\end{tabular}

P: fósforo; K: potássio; Na: sódio; Ca: cálcio; Mg: magnésio; Al: alumínio; MO: matéria orgânica; Zn: zinco; Fe: ferro; Mn: manganês; Cu: cobre; B: boro. 
elétrica (CE), turbidez (T), oxigênio dissolvido (OD), razão de adsorção de sódio (RAS), ferro reduzido $\left(\mathrm{Fe}^{2+}\right)$, ferro oxidado $\left(\mathrm{Fe}^{3+}\right)$, ferro total, sódio $(\mathrm{Na})$, cloro $(\mathrm{Cl})$, carbonato $\left(\mathrm{CO}_{3}^{2-}\right)$, dureza $\left(\mathrm{CaCO}_{3}\right)$, bicarbonato $\left(\mathrm{HCO}_{3}{ }^{2-}\right)$, sulfato $\left(\mathrm{SO}_{4}{ }^{2-}\right)$, fosfato $\left(\mathrm{PO}_{4}^{3-}\right)$, potássio $(\mathrm{K})$, cálcio $(\mathrm{Ca})$, magnésio $(\mathrm{Mg})$, boro $(\mathrm{B})$, manganês $(\mathrm{Mn})$, zinco $(\mathrm{Zn})$, cobre $(\mathrm{Cu})$, alumínio $(\mathrm{Al})$, nitrato $\left(\mathrm{NO}_{3}{ }^{-}\right)$e $\mathrm{DBO}$, conforme metodologia preconizada por Silva e Oliveira (2001).

Em função do grande número de parâmetros de qualidade medidos nos efluentes, foi aplicada uma técnica da estatística multivariada, a ACP, para avaliar a importância de cada parâmetro e promover a eliminação daqueles que contribuíram pouco em termos de variação da qualidade dos efluentes gerados. Para eliminar os efeitos de escala e unidades, foi feita a padronização dos dados, na qual os valores das variáveis são subtraídos de sua média e divididos pelo seu desvio padrão. Os parâmetros de qualidade selecionados na ACP estão apresentados na Tabela 2 .

A seleção do número de componentes extraídas neste trabalho seguiu os preceitos apresentados por Hair et al. (2005), em que se consideram somente aquelas com autovalor superior a um; dentro destas, foram escolhidas as variáveis que apresentaram coeficiente de correlação maior ou igual a 0,7 com as componentes selecionadas para comporem o IQ, conforme realizado por Bertossi et al. (2013a).

O IQ foi calculado por um valor numérico associado à qualidade individual de cada parâmetro $\left(\mathrm{q}_{\mathrm{i}}\right)$ elevada aos respectivos pesos desses parâmetros na avaliação da variabilidade total da qualidade do efluente $\left(\mathrm{w}_{\mathrm{i}}\right)$, como apresentado na Equação 1.

Tabela 2 - Limites dos parâmetros utilizados no índice de qualidade para cálculo da qualidade individual.

\begin{tabular}{|c|c|c|c|c|c|}
\hline \multirow{2}{*}{ Variáveis } & \multicolumn{5}{|c|}{$q_{i}$} \\
\hline & $80-100$ & $60-80$ & $40-60$ & $20-40$ & $0-20$ \\
\hline $\mathrm{CE}\left(\mathrm{dS} . \mathrm{m}^{-1}\right)$ & $\mathrm{O}, \mathrm{O}-0,7$ & $0,7-1,5$ & $1,5-3,0$ & $3,0-5,0$ & $>5,0$ \\
\hline $\mathrm{T}(\mathrm{UNT})$ & $0-30$ & $3-50$ & $50-70$ & 70-100 & $>100$ \\
\hline RAS & $0-3$ & $3-5$ & $5-7$ & $7-10$ & $>10$ \\
\hline $\mathrm{Na}\left(\right.$ meq. $\left.\mathrm{L}^{-1}\right)$ & $0,0-0,7$ & $0,7-1,5$ & $1,5-3,0$ & $3,0-5,0$ & $>5,0$ \\
\hline $\mathrm{Cl}\left(\right.$ meq. $\left.\mathrm{L}^{-1}\right)$ & $0-3$ & $3-5$ & $5-7$ & $7-10$ & $>10$ \\
\hline $\mathrm{CaCO}_{3}\left(\mathrm{mg} \cdot \mathrm{L}^{-1}\right)$ & $0-50$ & $50-150$ & $150-200$ & $200-300$ & $>300$ \\
\hline $\mathrm{HCO}_{3}^{2 \cdot}\left(\mathrm{mg} . \mathrm{L}^{-1}\right)$ & $0-3$ & $3-5$ & $5-7$ & $7-10$ & $>10$ \\
\hline $\mathrm{SO}_{4}{ }^{2 \cdot}\left(\right.$ meq. $\left.\mathrm{L}^{-1}\right)$ & $0-6$ & $6-10$ & $10-14$ & $14-20$ & $>20$ \\
\hline $\mathrm{PO}_{4}^{3-}\left(\mathrm{mg} \cdot \mathrm{L}^{-1}\right)$ & 0,0-1,0 & $1,0-1,5$ & $1,5-2,0$ & $2,0-3,0$ & $>3,0$ \\
\hline $\mathrm{K}\left(\mathrm{mg} \cdot \mathrm{L}^{-1}\right)$ & $0-10$ & $10-15$ & $15-20$ & $20-25$ & $>25$ \\
\hline Mg (meq. $\left.L^{-1}\right)$ & $0-2$ & $2-3$ & $3-5$ & $5-7$ & $>7$ \\
\hline$B\left(m g . L^{-1}\right)$ & $0-0,7$ & $0,7-1,5$ & $1,5-3$ & $3-5$ & $>5$ \\
\hline $\mathrm{NO}_{3}\left(\mathrm{mg} \cdot \mathrm{L}^{-1}\right)$ & $0-3$ & $3-5$ & $5-7$ & $7-10$ & $>10$ \\
\hline
\end{tabular}

CE: condutividade elétrica; T: turbidez; RAS: razão de adsorção de sódio; Na: sódio; $\mathrm{Cl}$ : cloro; $\mathrm{CaCO}_{3}$ dureza; $\mathrm{HCO}_{3}{ }^{2}$ : bicarbonato; $\mathrm{SO}_{4}{ }^{2}$ : sulfato; $\mathrm{PO}_{4}{ }^{3}$ : fosfato; $\mathrm{K}$ : potássio; $\mathrm{Mg}$ : magnésio; $\mathrm{B}$ : boro; $\mathrm{NO}_{3}$ : nitrato.
$I Q=\prod_{i=1}^{n} q i^{w i}$

Em que:

IQ = índice de qualidade do efluente (número entre 0 e 100);

$\mathrm{q}_{\mathrm{i}}=$ valor numérico associado à qualidade da i-ésima variável (número entre 0 e 100);

$\mathrm{w}_{\mathrm{i}}=$ peso correspondente a i-ésima variável (número entre 0 e 1).

Os valores de $\mathrm{q}_{\mathrm{i}}$ foram obtidos em função da concentração dos parâmetros medidos nos efluentes, na qual foram transformados em valores numéricos que representassem sua qualidade.

Os limites para o enquadramento dos valores de cada parâmetro no seu referido $\mathrm{q}_{\mathrm{i}}$ foram definidos considerando risco de salinidade, redução da infiltração de água no solo e problemas de toxicidade às plantas, seguindo os padrões de qualidade de água para irrigação proposto por Ayers e Westcot (1994). Na Tabela 2, estão apresentados os limites dos parâmetros que foram selecionados na ACP para comporem o IQ.

Para a obtenção do $\mathrm{q}_{\mathrm{i}}$ correspondente às concentrações dos parâmetros de qualidade medidos nos efluentes, utilizou-se a Equação 2, na qual se consideram o valor medido e os limites de $\mathrm{q}_{\mathrm{i}}$ das variáveis expressos na Tabela 2.

$q_{i}=q_{i \max }-\frac{\left(X_{i j}-X_{\text {inf }}\right)^{*} q_{\text {iamp }}}{X_{\text {amp }}}$

Em que:

$\mathrm{q}_{\mathrm{i} \text { máx }}=$ valor máximo de $\mathrm{q}_{\mathrm{i}}$ para a classe;

$\mathrm{X}_{\mathrm{ij}}=$ valor observado para a célula $\mathrm{ij}$;

$\mathrm{X}_{\mathrm{inf}}=$ limite inferior da classe a que pertence $\mathrm{X}_{\mathrm{ij}}$;

$\mathrm{q}_{\mathrm{i} \text { amp }}=$ amplitude de classe;

$\mathrm{X}_{\mathrm{amp}}=$ amplitude da classe a que pertence $\mathrm{X}_{\mathrm{ij}}$.

Para o cálculo de $\mathrm{X}_{\mathrm{amp}}$ da última classe para cada variável, foi considerado como limite superior o maior valor encontrado nas análises físico-químicas das amostras de efluente para cada variável, conforme realizado por Andrade et al. (2005).

Para atribuir os pesos $\left(\mathrm{w}_{\mathrm{i}}\right)$ a cada parâmetro de qualidade de efluente utilizado no IQ, foram tomados como base os resultados obtidos na ACP. Nesse procedimento, foram utilizados os autovalores das componentes e a contribuição de cada variável pelas respectivas componentes, extraídas da ACP, como alcançado por Andrade et al. (2005).

Inicialmente, calculou-se o somatório dos autovalores de todas as componentes multiplicados pela contribuição de cada variável. Em seguida, foram ponderados os valores de $\mathrm{w}_{\mathrm{i}}$ para cada parâmetro em função de sua contribuição por cada componente, de forma que a soma de todos os $\mathrm{w}_{\mathrm{i}}$ fosse igual a um, conforme demonstrado na Equação 3.

$W_{i}=\frac{F_{1} P_{1 i}+F_{2} P_{2 i}}{\left(\sum_{j=1}^{n} F_{1} P_{1 j}\right)+\left(\sum_{j=1}^{n} F_{2} P_{2 j}\right)}$ 
Em que:

$\mathrm{F}_{1}$ e $\mathrm{F}_{2}=$ autovalores das componentes principais;

$\mathrm{P}_{1}$ e $\mathrm{P}_{2}=$ contribuições das variáveis pelas componentes principais;

i e $\mathrm{j}$ = índices para as variáveis;

$\mathrm{n}$ = número de variáveis envolvidas na ACP.

De posse dos valores de $\mathrm{q}_{\mathrm{i}}$ e $\mathrm{w}_{\mathrm{i}}$, determinou-se a qualidade dos efluentes bruto e tratado, segundo os parâmetros avaliados, para fertirrigação, a fim de evitar problemas de salinidade e sodicidade no solo, que, indicada pelo IQ em uma escala de 0 a 100, foi classificada segundo a Tabela 3 .

As características mais importantes para variação da qualidade dos efluentes selecionadas na ACP foram utilizadas para o estudo da semelhança entre o efluente bruto aplicado no sistema e o tratado, que foi realizado pela AAHC.

As amostras de efluente doméstico foram agrupadas com base na similaridade das características analisadas, definida pela variação entre os coeficientes de agrupamento de dois grupos consecutivos, conforme descrito por Hair et al. (2005). Como as variáveis utilizadas neste estudo são reais, adotou-se a combinação da distância euclidiana ao quadrado para a formação da matriz de similaridade e o algoritmo do método de ligação Ward, conforme realizado por vários autores, como Fernandes et al. (2010), Palácio et al. (2009) e Bertossi et al. (2013b).

\section{RESULTADOS E DISCUSSÃO}

Pela análise estatística multivariada, um modelo com duas componentes foi adequado para representar as variáveis químicas analisadas no efluente e explicar aproximadamente $90,54 \%$ da variância total. A primeira e a segunda componentes principais explicaram, respectivamente, 72,77 e $17,77 \%$ da variância total dos dados, concentrando, em duas dimensões, 90,54\% das informações antes diluídas em 25 dimensões (número de variáveis analisadas). Resultados semelhantes foram encontrados por Andrade et al. (2007b), ao avaliarem a qualidade das águas superficiais da bacia hidrográfica do Alto Acaraú, no Ceará, Brasil, e por Bertossi et al. (2013a), ao avaliarem a qualidade da água em microbacias com diferentes coberturas do solo, no Sul do Espírito Santo, Brasil.

Já Meireles et al. (2010), ao utilizarem a ACP para avaliação da qualidade da água para irrigação do rio Acaraú, no Ceará, Brasil, selecionaram três componentes indicadoras da qualidade das águas superficiais estudadas, que, juntas, explicaram $72,3 \%$ da variância total. No presente trabalho, foram encontrados valores superiores, ou seja, com apenas duas componentes, foi possível explicar 90,54\% da variância total dos dados.

Na primeira componente, foram selecionadas as variáveis $\mathrm{CE}, \mathrm{T}$, RAS, $\mathrm{Na}, \mathrm{Cl}, \mathrm{HCO}_{3}^{2-}, \mathrm{SO}_{4}^{2-}, \mathrm{PO}_{4}^{3-}, \mathrm{K}, \mathrm{B} \mathrm{e} \mathrm{NO}_{3}^{-}$; e, na segunda, as variáveis dureza $\left(\mathrm{CaCO}_{3}\right)$ e $\mathrm{Mg}$. Meireles et al. (2010), ao utilizarem a técnica das componentes principais para selecionarem as características mais importantes das águas superficiais do rio Acaraú, no Ceará, Brasil, para uso na irrigação, obtiveram resultados semelhantes aos obtidos neste trabalho. Os autores supracitados selecionaram as variáveis: $\mathrm{CE}$, $\mathrm{Mg}, \mathrm{Na}, \mathrm{K}, \mathrm{Cl}, \mathrm{HCO}_{3}, \mathrm{RAS}, \mathrm{PO}_{4}, \mathrm{NH}_{4}, \mathrm{NO}_{3} \mathrm{e} \mathrm{SO}_{4}$.

Portanto, a qualidade para irrigação/fertirrigação está relacionada aos sais solúveis presentes, demonstrando serem essas as características mais importantes para avaliação da qualidade de água/efluente para tal uso. Segundo Silva et al. (2011), altos níveis de sais nas águas

Tabela 3 - Classificação do índice de qualidade de água para irrigação, segundo Meireles et al. (2010).

\begin{tabular}{|c|c|c|c|}
\hline \multirow{2}{*}{ IQ } & \multirow{2}{*}{$\begin{array}{l}\text { Restrições } \\
\text { ao uso }\end{array}$} & \multicolumn{2}{|c|}{ Recomendações } \\
\hline & & Solo & Planta \\
\hline $85 \leq 100$ & $\begin{array}{l}\text { Nenhuma } \\
\text { restrição }\end{array}$ & $\begin{array}{l}\text { Pode ser usado para a maioria dos solos com baixa } \\
\text { probabilidade de causar problemas de salinidade e sodicidade, } \\
\text { sendo recomendada lixiviação em conjunto com práticas de } \\
\text { irrigação, exceto em solos com baixa permeabilidade. }\end{array}$ & Não há risco de toxicidade para a maioria das plantas. \\
\hline $70 \leq 85$ & $\begin{array}{l}\text { Baixa } \\
\text { restrição }\end{array}$ & $\begin{array}{c}\text { Recomendado o uso em solos com textura leve ou moderada } \\
\text { permeabilidade, sendo recomendada a lixiviação de sal. Em solos } \\
\text { muito argilosos, podem ocorrer problemas de sodicidade. Deve } \\
\text { ser evitado o uso em solos com altos níveis de argila 2:1. }\end{array}$ & Devem-se evitar plantas sensíveis ao sal. \\
\hline $55 \leq 70$ & $\begin{array}{l}\text { Moderada } \\
\text { restrição }\end{array}$ & $\begin{array}{l}\text { Pode ser usada em solos com moderada a alta permeabilidade, } \\
\text { sendo sugerida lixiviação moderada de sais. }\end{array}$ & $\begin{array}{l}\text { Plantas com tolerância moderada } \\
\text { a sais podem ser cultivadas. }\end{array}$ \\
\hline $40 \leq 55$ & $\begin{array}{l}\text { Alta } \\
\text { restrição }\end{array}$ & $\begin{array}{l}\text { Pode ser usado em solos com alta permeabilidade, sem camadas } \\
\text { compactas. Cronograma de irrigação de alta frequência deve ser } \\
\text { adotado para água com CE acima } 2 \mathrm{dS} \cdot \mathrm{m}^{-1} \text { e RAS acima de } 7 \text {. }\end{array}$ & 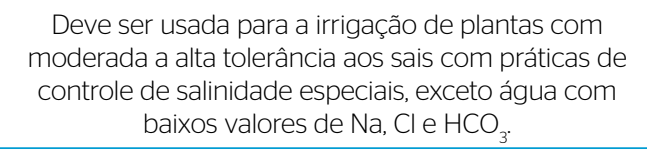 \\
\hline $0 \leq 40$ & $\begin{array}{l}\text { Severa } \\
\text { restrição }\end{array}$ & $\begin{array}{c}\text { Deve ser evitada a sua utilização para a irrigação, } \\
\text { em condições normais. Em casos especiais, pode ser } \\
\text { utilizada de vez em quando. Agua com baixos níveis de } \\
\text { sal e RAS alta exigem aplicação de gesso. }\end{array}$ & $\begin{array}{l}\text { Apenas as plantas com tolerância elevada a sal, com } \\
\text { exceção de águas com baixíssimos valores de } \mathrm{Na}, \mathrm{Cl} \text { e } \\
\mathrm{HCO}_{3} \text { devem ser cultivadas nesse solo. }\end{array}$ \\
\hline
\end{tabular}

IQ: índice de qualidade; CE: condutividade elétrica; RAS: razão de adsorção de sódio; Na: sódio; Cl: Cloro; $\mathrm{HCO}_{3}$; bicarbonato. 
de irrigação tanto são prejudiciais ao desenvolvimento das culturas como causam problemas no solo e obstrução de tubulações e emissores dos sistemas de irrigação.

A ACP promoveu boa redução no número de variáveis, na qual foram selecionadas 13 das 25 variáveis analisadas no efluente, ou seja, foram selecionadas $50 \%$ das variáveis analisadas para comporem o IQ. Da mesma forma, Bertossi et al. (2013a) obtiveram bons resultados ao aplicarem a técnica das componentes principais para selecionarem as variáveis mais representativas da qualidade da água de microbacias com diferentes usos do solo no sul do Espírito Santo, na qual foram selecionadas sete das 13 variáveis analisadas, ou seja, foram selecionadas aproximadamente $50 \%$ das variáveis para comporem o IQ, assim como no presente estudo.

Já Andrade et al. (2007a), com o objetivo de identificarem a origem dos fatores que determinavam a qualidade das águas na parte baixa da Bacia do Acaraú (Ceará, Brasil), não obtiveram bons resultados ao aplicar a ACP para reduzir o número de variáveis, uma vez que o melhor ajuste do modelo ocorreu com a inclusão de 18 das 25 variáveis analisadas.

A concentração das variáveis selecionadas na ACP para comporem o IQ foi transformada em valores numéricos que representassem sua qualidade $\left(\mathrm{q}_{\mathrm{i}}\right)$. Os valores de $\mathrm{q}_{\mathrm{i}}$ para cada variável química, variando de 0 a 100, estão apresentados na Tabela 4.

Para a maioria dos efluentes testados, os valores de $\mathrm{q}_{\mathrm{i}}$ foram menores para o sódio, a dureza, o potássio e o nitrato, o que sugere que essas são as características que mais diferenciaram um efluente do outro, afetando de forma significativa sua qualidade, reduzindo-a.

Tabela 4 - Valores de qualidade individual para os efluentes domésticos bruto e tratado.

\begin{tabular}{|c|c|c|c|c|c|c|c|}
\hline \multirow{2}{*}{ Variáveis } & \multicolumn{2}{|c|}{ Azevém } & \multicolumn{2}{|c|}{ Aveia preta } & \multicolumn{2}{|c|}{ Centeio } & \multirow{2}{*}{ EDB } \\
\hline & 50 & 100 & 50 & 100 & 50 & 100 & \\
\hline $\begin{array}{l}\text { Condutividade } \\
\text { elétrica }\end{array}$ & 72,73 & 81,57 & 73,98 & 84,97 & 71,13 & 82,77 & 60,00 \\
\hline Turbidez & 86,94 & 96,02 & 89,21 & 96,91 & 92,61 & 96,18 & 37,50 \\
\hline RAS & 73,88 & 91,50 & 78,64 & 93,32 & 75,26 & 90,85 & 62,00 \\
\hline Sódio & 32,64 & 57,33 & 38,16 & 67,90 & 38,00 & 58,13 & 24,40 \\
\hline Cloro & 97,06 & 98,55 & 97,31 & 98,76 & 96,75 & 98,79 & 96,13 \\
\hline Dureza & 67,59 & 48,07 & 68,50 & 60,37 & 65,58 & 61,03 & 64,43 \\
\hline Bicarbonato & 99,15 & 94,20 & 99,17 & 95,91 & 99,21 & 95,94 & 95,60 \\
\hline Sulfato & 96,11 & 96,38 & 95,85 & 96,77 & 95,94 & 96,95 & 98,13 \\
\hline Fosfato & 95,74 & 96,72 & 94,18 & 96,30 & 93,86 & 96,22 & 39,40 \\
\hline Potássio & 8,23 & 49,20 & 4,84 & 64,20 & 6,80 & 38,80 & 0,71 \\
\hline Magnésio & 96,16 & 95,62 & 95,70 & 95,67 & 95,58 & 96,78 & 93,90 \\
\hline Boro & 96,43 & 98,89 & 95,40 & 98,33 & 96,14 & 98,91 & 95,43 \\
\hline Nitrato & 5,27 & 83,67 & 8,07 & 75,00 & 2,86 & 61,00 & 3,20 \\
\hline
\end{tabular}

EDB: efluente doméstico bruto; RAS: razão de adsorção de sódio.
Altas concentrações de Na na água de irrigação podem interferir nos atributos físicos do solo, causando dispersão da argila, entupimento dos poros e redução da permeabilidade (MUYEN; MOORE; WRIGLEY, 2011). Águas com alta dureza podem formar incrustações nas paredes das tubulações e emissores restringindo a passagem da água (BERNARDO; SOARES; MANTOVANI, 2006). A utilização de águas para irrigação com concentrações de $\mathrm{NO}_{3}^{-}$e $\mathrm{K}$ acima dos limites aceitáveis durante um longo período podem ocasionar distúrbios nutricionais na cultura irrigada (HANJRA et al., 2012). Além disso, o $\mathrm{NO}_{3}$, por ser íon móvel no solo, pode vir a contaminar os aquíferos subterrâneos utilizados para abastecimento de água (PINTO et al., 2013).

Já o EDB, além dessas características, apresentou baixo $\mathrm{q}_{\mathrm{i}}$ para a variável $\mathrm{T}$, demonstrando ser esta a principal característica que diferenciou os efluentes tratados do bruto. Taebi e Droste (2008), ao utilizarem a técnica do escoamento superficial para tratamento de efluentes, observaram ótima remoção de turbidez, podendo atingir valores em torno de $70 \%$.

A depuração do efluente no sistema de tratamento por escoamento superficial dá-se à medida que o efluente escoa na área recoberta pela vegetação, onde os sólidos em suspensão são filtrados (TONETTI; COURACCI FILHO; STEFANUTTI, 2012). Segundo Surampalli et al. (2007), essa técnica também apresenta ótima remoção de sólidos em suspensão, podendo variar de 51 a 92\%, sendo esta a principal característica responsável pela alta turbidez do efluente, o qual, se utilizado para irrigação, pode causar entupimento das tubulações, reduzindo a área de condução de água e queda na eficiência do sistema (AYERS; WESTCOT, 1994).

Os valores do peso $\mathrm{w}_{\mathrm{i}}$ não variaram muito entre as variáveis, sendo maior para o $\mathrm{Cl}$, a $\mathrm{CE}$, a RAS, o $\mathrm{K}$, o Na, a dureza $\left(\mathrm{CaCO}_{3}\right)$, o $\mathrm{HCO}_{3}{ }^{2-}$, $\mathrm{o} \mathrm{SO}_{4}{ }^{2-}, \mathrm{o} \mathrm{Mg}$ e o $\mathrm{B}$, apresentando o índice alta sensibilidade à variabilidade dessas características. Já as variáveis $\mathrm{T}$ e $\mathrm{PO}_{4}^{3-}$ obtiveram pesos inferiores aos demais (Tabela 5).

Procedimento semelhante foi adotado por Meireles et al. (2010) ao utilizarem a técnica da ACP a fim de desenvolver um IQ de água para irrigação. Os sobreditos autores encontraram maiores valores do peso $\mathrm{w}_{\mathrm{i}}$ para as variáveis $\mathrm{CE}, \mathrm{Na}, \mathrm{HCO}_{3}^{2-}, \mathrm{Cl}$ e RAS, o que corrobora os resultados encontrados neste trabalho, atestando a importância do monitoramento de sais na água de irrigação.

De posse dos valores de $\mathrm{q}_{\mathrm{i}}$ e $\mathrm{w}_{\mathrm{i}}$, foi possível calcular o IQ para os efluentes e classificá-los de acordo com os limites para uso na irrigação

Tabela 5 - Pesos $\left(w_{i}\right)$ para as variáveis do índice de qualidade.

\begin{tabular}{c|c|c|c|c|c|c|c} 
& CE & T & RAS & Na & Cl & Dureza & Bicarbonato \\
W $_{i}$ & 0,082 & 0,066 & 0,082 & 0,081 & 0,083 & 0,081 & 0,078 \\
& Sulfato & Fosfato & $\mathrm{K}$ & $\mathrm{Mg}$ & $\mathrm{B}$ & Nitrato & \\
\hline $\mathrm{W}_{\mathrm{i}}$ & 0,078 & 0,062 & 0,082 & 0,076 & 0,076 & 0,073 & \\
\hline
\end{tabular}

CE: condutividade elétrica; T: turbidez; RAS: razão de adsorção de sódio; Na: sódio; Cl: cloro; K: potássio; Mg: magnésio; B: boro. 
apresentados na Tabela 3. De acordo com a Tabela 6, pode-se constatar que os valores de IQ para os efluentes tratados variaram entre 51,45 e 84,85 (classe de alta a baixa restrição). Já o efluente bruto apresentou valor de IQ de 35,85 (classe de restrição severa).

Os efluentes com alta restrição devem ser utilizados para a irrigação de plantas com moderada a alta tolerância aos sais e em solos com alta permeabilidade. Os efluentes com baixa restrição devem ser utilizados em solos com textura leve ou moderada permeabilidade. Já o efluente bruto que apresentou restrição severa deve ser evitado para uso na irrigação em condições normais, sem adoção de práticas de controle dos problemas relacionados à salinidade e à sodicidade.

Apesar de neste estudo de qualidade ter sido utilizado efluente doméstico, resultados semelhantes foram obtidos por Meireles et al. (2010) ao avaliarem a qualidade das águas do rio Acaraú, no Ceará, Brasil, para irrigação. Esses autores também observaram que a qualidade das águas estudadas variou de baixa a severa restrição, o que sugere que tanto água quanto efluente, se utilizados de forma inadequada, podem causar problemas ao solo e à cultura irrigada por conta das suas características químicas e, principalmente, se contivessem altos teores de sais.

$\mathrm{Na}$ Figura 1, apresenta-se o dendograma referente à AAHC, no qual as amostras de efluentes domésticos bruto e tratado foram agrupadas quanto à similaridade das características de qualidade selecionadas como mais importantes na ACP. Observa-se que o ponto ótimo de corte para formação dos grupos de similaridade se encontra na distância de $20 \%$, pois, a partir deste, ocorre o distanciamento maior na medida de similaridade para a formação dos grupos. Dessa forma, definem-se três agrupamentos distintos.

Percebe-se que dois dos grupos formados foram compostos, cada um, de efluentes tratados, nas diferentes plantas utilizadas (AZ, AV e C) no sistema de tratamento por escoamento superficial, o que os diferenciou foi a taxa de aplicação (50 e 100 kg.ha-1. dia-1 de DBO). Já o EDB ficou em um grupo sozinho, o que significa que apresentou características diferentes dos efluentes tratados.

Os resultados obtidos no agrupamento são condizentes com o IQ, no qual todas as amostras de efluente tratado utilizando as diferentes

Tabela 6 - Índice de qualidade dos efluentes domésticos bruto e tratado.

\begin{tabular}{l|c|c|c}
\multirow{2}{*}{ Plantas } & Taxa & IQ & Classificação \\
\cline { 2 - 4 } & 50 & 54,02 & Alta restrição \\
\cline { 2 - 4 } & 100 & 80,4 & Baixa restrição \\
\hline \multirow{2}{*}{ Aveia preta } & 50 & 54,4 & Alta restrição \\
\cline { 2 - 4 } & 100 & 84,85 & Baixa restrição \\
\hline \multirow{2}{*}{ Centeio } & 50 & 51,45 & Alta restrição \\
\cline { 2 - 4 } & 100 & 79,1 & Baixa restrição \\
\hline Efluente doméstico bruto & & 35,85 & Severa restrição \\
\hline
\end{tabular}

IQ: índice de qualidade. espécies de forrageiras e uma taxa de aplicação de 50 kg.ha-1. dia $^{-1}$ de DBO apresentaram valores do IQ próximos e com a mesma classificação (alta restrição) e, na análise de agrupamento, ficaram no mesmo grupo. As amostras de efluente tratado utilizando uma taxa de aplicação de $100 \mathrm{~kg} \cdot \mathrm{ha}^{-1} \cdot \mathrm{dia}^{-1}$ também apresentaram valores bem próximos do IQ, com a mesma classificação (baixa restrição), e formaram um grupo distinto. Já o EDB apresentou um valor de desse índice bem abaixo das amostras de efluente tratado, apresentando uma severa restrição ao uso na irrigação e, por isso, na análise de agrupamento, ficou sozinho.

Do mesmo modo, os resultados obtidos com o IQ e com a análise de agrupamento demostram que a técnica do escoamento superficial foi eficiente no tratamento do EDB, o qual passou de uma severa (EDB) para alta e baixa restrição ao uso na fertirrigação (efluentes tratados). Além disso, pode-se observar que as plantas utilizadas no experimento não influenciaram na qualificação, nem no agrupamento dos efluentes tratados, que diferenciaram apenas quanto às taxas de aplicação utilizadas.

Segundo Wen et al. (2007), a espécie de planta utilizada nas rampas de tratamento por escoamento superficial pode influenciar na eficiência do sistema. Esses autores, ao avaliarem a remoção de poluentes por quatro espécies de gramíneas diferentes utilizadas nesse tipo de tratamento - Paragrass (Brachairia mutica), Nilegrass (Acroceras macrum), Cattail (Typha oricntalis) e Vetiver (Vetiveria zizanioides) observaram diferentes remoções para as diferentes espécies de plantas utilizadas. Porém, cabe salientar que, no presente estudo, foram utilizadas outras espécies de plantas, as quais influenciaram, de forma semelhante, no tratamento do efluente doméstico.

Apenas a taxa de aplicação interferiu na eficiência do sistema de tratamento, sendo observada melhor qualidade nos efluentes tratados na taxa de $100 \mathrm{~kg} \cdot \mathrm{ha}^{-1} \cdot \mathrm{dia}^{-1}$ de DBO. Assim como no presente estudo,

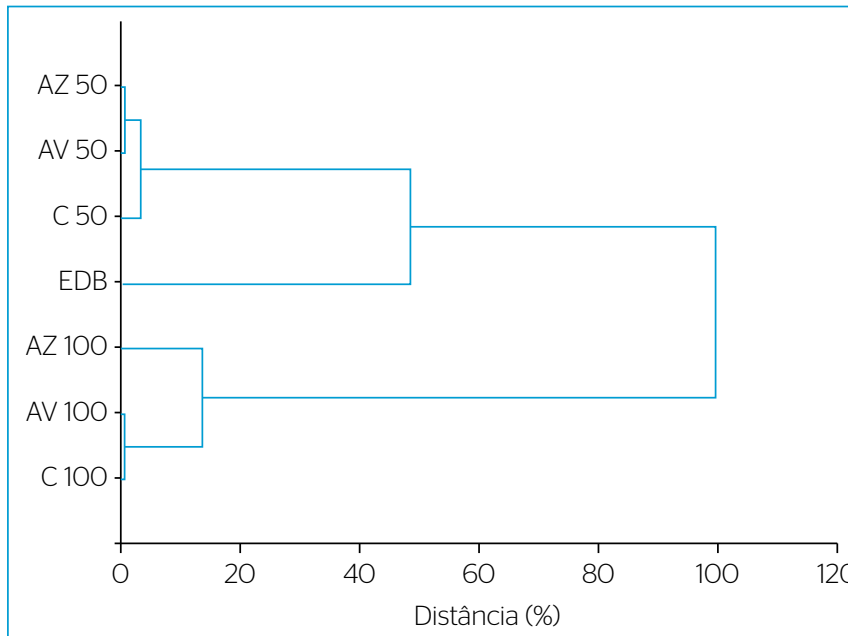

AZ: azevém; AV: aveia preta; C: centeio; 50 e 100: taxas de aplicação de efluente em kg.ha-1.dia' de demanda bioquímica de oxigênio; EDB: efluente doméstico bruto

Figura 1 - Dendrograma dos efluentes agrupados quanto às características de qualidade selecionadas na análise de componentes principais para comporem o índice de qualidade. 
diversos autores, ao utilizarem a técnica do escoamento superficial para tratamento de efluentes, observaram que diferentes taxas de aplicação proporcionaram diferenças na eficiência do tratamento (LOURES et al., 2006; TAEBI; DROSTE, 2008; TONETTI; COURACCI FILHO; STEFANUTTI, 2012).

Dessa forma, os resultados obtidos sugerem que a taxa de aplicação de efluente no sistema de tratamento por escoamento é mais importante que a espécie de planta utilizada, sendo necessário determinar, de forma criteriosa, a melhor taxa a ser utilizada.

\section{CONCLUSÕES}

Os resultados apresentados neste trabalho conduziram às seguintes conclusões:
- As variáveis mais representativas da variabilidade da qualidade dos efluentes analisados foram: $\mathrm{CE}, \mathrm{T}, \mathrm{RAS}, \mathrm{Na}, \mathrm{Cl}, \mathrm{HCO}_{3}{ }^{2-}, \mathrm{SO}_{4}{ }^{2-}$, $\mathrm{PO}_{4}^{3-}, \mathrm{K}, \mathrm{B}, \mathrm{NO}_{3}^{-}$, dureza $\left(\mathrm{CaCO}_{3}\right)$ e $\mathrm{Mg}$;

- O IQ para os efluentes tratados apresentaram de alta a baixa restrição ao uso na fertirrigação, enquanto o efluente bruto demonstrou restrição severa;

- A técnica do escoamento superficial foi eficiente no tratamento do esgoto doméstico bruto;

- A AAHC formou três grupos distintos de qualidade, que diferiram quanto à taxa de aplicação de efluente utilizada no sistema de tratamento;

- As plantas utilizadas no experimento não influenciaram na qualificação dos efluentes tratados nem na segregação dos grupos formados.

\section{REFERÊNCIAS}

ANDRADE, E.M.; ARAÚJO, L.F.P.; ROSA, M.F.; DISNEY, W.; ALVES, A.B. (2007a) Seleção dos indicadores da qualidade das águas superficiais pelo emprego da análise multivariada. Engenharia Agrícola, Jaboticabal, v. 27, n. 3. http://doi.org/10.1590/S010069162007000400011

ANDRADE, E.M.; ARAÚJO, L.F.P.; ROSA, M.F.; GOMES, R.B.; LOBATO, F.A.O. (2007b) Fatores determinantes da qualidade das águas superficiais na bacia do Alto Acaraú, Ceará, Brasil. Ciência Rural, Santa Maria, v. 37, n. 6, p. 1791-1797. http://dx.doi.org/10.1590/SO10384782007000600045

ANDRADE, E.M.; PALÁCIO, H.A.Q.; CRISÓSTOMO, L.A.; SOUZA, I.H.; TEIXEIRA, A.S. (2005) Índice de qualidade de água, uma proposta para o vale do rio Trussu, Ceará. Revista Ciência Agronômica, Fortaleza, v. 36, n. 2, p. 135-142.

ARDILA, A.N.; SALDARRIAGA, J.C. (2013) Formulación de un Índice Global de Calidad de Aguas Residuales para Riego. Avances en Ciencias e Ingeniería, v. 4, n. 3, p. 11-26.

AYERS, R.S.; WESTCOT, D.W. (1994) Water Quality for Agriculture. Roma: Food and Agriculture Organization of the United Nations. (Serie: FAO Irrigation and Drainage Papers, n० 29). Disponível em <http://www.fao.org/DOCREP/O03/TO234E/TO234EOO.htm\#TOC>. Acesso em: 10 mar. 2014.

BERNARDO, S.; SOARES, A.A.; MANTOVANI, E.C. (2006) Manual de Irrigação. Viçosa: Editora UFV. 625 p.

BERTOSSI, A.P.A.; CECÍLIO, R.A.; NEVES, M.A.; GARCIA, G.O. (2O13a) Qualidade da água em microbacias hidrográficas com diferentes coberturas do solo no sul do Espírito Santo. Revista Árvore, v. 37, n. 1, p. 107-117. http://dx.doi.org/10.1590/S0100-67622013000100012
BERTOSSI, A.P.A.; MENEZES, J.P.C.; CECÍLIO, R.A.; GARCIA, G.O. NEVES, M.A. (2013b) Seleção e agrupamento de indicadores da qualidade de águas utilizando Estatística Multivariada. Semina: Ciências Agrárias, Londrina, v. 34, n. 5, p. 2025-2036. http://dx.doi org/10.5433/1679-0359.2013v34n5p2025

COSTA, D.M.A.; BARROS JÚNIOR, A.C. (2005) Avaliação da Necessidade do Reúso de Águas Residuais. Holos, v. 2, p. 82. https:// doi.org/10.15628/holos.2005.74

EMPRESA BRASILEIRA DE PESQUISA AGROPECUÁRIA (EMBRAPA). (2009) Manual de análises químicas de solos, plantas e fertilizantes. Brasília: Embrapa Comunicação para Transferência de Tecnologia. $627 \mathrm{p}$.

FERNANDES, F.B.P.; ANDRADE, E.M.; FONTENELE, S.B.; MEIRELES, A.C.M.; RIBEIRO, J.A. (2010) Análise de agrupamento como suporte à gestão qualitativa da água subterrânea no semiárido cearense. RevistaAgro@mbiente On-line, Boa Vista, v. 4, n. 2, p. 86-95. http:// dx.doi.org/10.18227/1982-8470ragro.v4i2.402

FLORES, J.C. (2002) Comments on the use of water quality indices to verify the impact of Cordoba City (Argentina) on Suquia River Water Research, v. 36, n. 18, p. 4664-4666. http://dx.doi.org/10.1016/ s0043-1354(02)00181-1

HAIR, J.J.F.; ANDERSON, R.E.; TATHAM, R.L.; BLACK, W.C. (2005) Análise multivariada de dados. 5. ed. Porto Alegre: Bookman. 593 p.

HANJRA, M.A.; BLACKWELL, J.; CARR, G.; ZHANG, F.; JACKSON, T.M. (2012) Wastewater irrigation and environmental health: Implications for water governance and public policy. International Journal of Hygiene and Environmental Health, v. 215, n. 3, p. 255 269. https://doi.org/10.1016/j.ijheh.2011.10.003 
HURLEY, T.; SADIQ, R.; MAZUMDER, A. (2012) Adaptation and evaluation of the Canadian Council of Ministers of the Environment Water Quality Index (CCME WQI) for use as an effective tool to characterize drinking source water quality. Water Research, v. 46, n. 11, p. 3544-3552. https://doi.org/10.1016/j. watres.2012.03.061

LOURES, A.P.S.; SOARES, A.A.; MATOS, A.T.; CECON, P.R.; PEREIRA, O.G. (2006) Remoção de fósforo em sistema de tratamento de esgoto doméstico, por escoamento superficial. Revista Brasileira de Engenharia Agrícola e Ambiental, Campina Grande, v. 10, n. 3, p. 706-714. http://dx.doi.org/10.1590/S141543662006000300025

MEIRELES, A.C.M.; ANDRADE, E.M.; CHAVES, L.C.G.; FRISCHKORN, H.; CRISOSTOMO, L.A. (2010) A new proposal of the classification of irrigation water. Revista Ciência Agronônica, v. 41, n. 3, p. 349-357.

MUYEN, Z:; MOORE, G.A.; WRIGLEY, R.J. (2011) Soil salinity and sodicity effects of wastewater irrigation in South East Australia. Agricultural Water Management, v. 99, n. 1, p. 33-41. http://dx.doi. org/10.1016/j.agwat.2011.07.021

PALÁCIO, H.A.Q.; ANDRADE, E.M.; LOPES, F.B.; ALEXANDRE, D.M.B.; ARRAES, F.D.D. (2009) Similaridade da qualidade das águas superficiais da bacia do Curu, Ceará. Ciência Rural, Santa Maria, v. 39, n. 9, p. 2494-2500. http://dx.doi.org/10.1590/S010384782009000900017

PINTO, M.C.K.; CRUZ, R.L.; FRIGO, E.P.; FRIGO, M.S.; HERMES, E. (2O13) Contaminação das águas subterrâneas por nitrogênio devido à irrigação com efluente do tratamento de esgoto. Irriga, Botucatu, v. 18, n. 2, p. 270-281. https://doi.org/10.15809/irriga.2013v18n2p270
SILVA, I.N.; FONTES, L.O.; TAVELLA, L.B.; OLIVEIRA, J.B.; OLIVEIRA, A.C. (2011) Qualidade de água na irrigação. Agropecuária Científica no Semi-Árido, v. 7, n. 3, p. 1-15. http://dx.doi.org/10.30969/acsa.v7i3.134

SILVA, S.A.; OLIVEIRA, R. (2001) Manual de análises físico-química de águas de abastecimento e residuárias. Marcone: Capina Grande. 266 p.

SURAMPALLI, R.Y.; BANERJI, S.K.; TYAGI, R.D.; YANG, P.Y. (2007) Integrated advanced natural wastewater treatment system for small communities. Water Science \& Technology, v. 55, n. 11, p. 239 243. http://dx.doi.org/10.2166/wst.2007.371

TAEBI, A.; DROSTE, R.L. (2008) Performance of an overland flow system for advanced treatment of wastewater plant effluent. Journal of Environmental Management, v. 88, n. 4, p. 688-696. http://dx.doi.org/10.1016/j.jenvman.2007.03.038

TONETTI, A.L.; COURACCI FILHO, B.; STEFANUTTI, R. (2012) Póstratamento de efluente de filtros anaeróbios operados com baixo tempo de detenção hidráulica por escoamento superficial no solo. Engenharia Sanitária e Ambiental, v. 17, n. 1, p. 7-12. http://dx.doi. org/10.1590/S1413-41522012000100004

WANDA, E.M.M.; GULULA, L.C.; PHIRI, G. (2012) Determination of characteristics and drinking water quality index in Mzuzu City, Norther Malawi. Physics and Chemistry Earth, v. 50-52, p. 92-97. http://dx.doi.org/10.1016/j.pce.2012.09.004

WEN, C.G.; CHEN, T.H.; HSU, F.H.; LU, C.H.; LIN, J.B.; CHANG, C.H.; CHANG, S.P.; LEE, C.S. (2007) A high loading overland flow system Impacts on soil characteristics, grass constituents, yields and nutrient removal. Chemosphere, v. 67, n. 8, p. 1588-1600. https://doi. org/10.1016/j.chemosphere.2006.11.053 